\title{
SITUAÇÃO EPIDEMIOLÓGICA DO TRACOMA NO PIAUÍ, NORDESTE BRASILEIRO
}

\section{Miguel Henrique Pereira de Paiva}

Discente de enfermagem pela Faculdade Integral Diferencial com período sanduíche em Walsh University.

E-mail: miguelhppaiva@hotmail.com

\section{Maria Amélia de Oliveira Costa}

Mestre em Enfermagem. Docente da Universidade Estadual do Piauí e Faculdade Integral Diferencial Brasil.

\section{Rogério Ferreira Luz}

Especialista em docência do ensino superior e em saúde da família, Brasil.

\section{Renato Douglas e Silva Souza}

Mestre em farmacologia. Enfermeiro na Empresa Brasileira de Serviços Hospitalares, Brasil.

Adelmo Barbosa de Miranda Junior

Especialista em urgência e emergência. Enfermeiro na Empresa Brasileira de Serviços Hospitalares, Brasil.

Francisco Rogério de Araújo Melo Filho

Discente de Medicina pela Faculdade Integral Diferencial, Brasil.

\section{Mayara Rafaela dos Reis}

Enfermeira no Hospital de Urgência de Teresina, Brasil.
RESUMO: Este estudo objetivou apresentar a epidemiologia do tracoma no Piauí com enfoque no número de casos positivos, formas clínicas e locais de ocorrência. Tratou-se de um estudo exploratório de abordagem quantitativa, realizado a partir da análise de dados de acesso restrito sobre a situação epidemiológica do tracoma no Piauí. Casos positivos de tracoma foram notificados em 34 municípios piauienses nos últimos nove anos, a maioria localizada na região Sudoeste do Estado e apresentando baixo índice de desenvolvimento humano. O município de Uruçuí apresentou o maior número de casos. foram notificados 593 casos de tracoma, sendo o tracoma folicular a forma clínica mais frequente, além de casos raros de tracoma inflamatório intenso e um caso de tracoma cicatricial. Considerando dados do último inquérito nacional do tracoma no Piauí, houve redução de municípios afetados, corroborando com a meta da Organização Mundial de Saúde de eliminação de tracoma até 2020.

PALAVRAS-CHAVE: Tracoma; Epidemiologia; Prevalência; Inquéritos epidemiológicos; Promoção da saúde.

\section{EPIDEMIOLOGICAL SITUATION OF TRACHOMA IN THE NORTHEASTERN STATE OF PIAUÍ, BRAZIL}

ABSTRACT: Trachoma epidemiology in the Brazilian state of Piauí is provided, with special emphasis on the number of positive cases, clinical types and sites of occurrence. Current exploratory and quantitative analysis has been undertaken by analyzing restricted data on the epidemiological situation of trachoma in Piauí. Positive trachoma cases were notified in 34 municipalities during the last nine years, most of which from the southeastern region of the state with low human development rates. The municipality of Uruçuí had the highest number of cases. Further, 593 cases of trachoma, particularly follicle mode, were notified, even though there were rare cases of intense inflammatory trachoma and one case of cicatricial trachoma. When data on the latest national survey on trachoma in Piauí are investigated, one may note a decrease in the municipalities affected, corroborating with the aim of WHO for its elimination by 2020 .

KEY WORDS: Trachoma; Epidemiology; Prevalence; Epidemiological surveys; Health promotion. 


\section{INTRODUÇÃO}

O tracoma é uma infecção inflamatória ocular que tem como agente etiológico a bactéria gram-negativa Chlamydiatrachomatis. Esta continua a ser a principal causa infecciosa de cegueira no mundo, sendo as principais características das zonas endêmicas as más condições sanitárias e as habitações lotadas em comunidades pobres de recursos (AAO, 2016; ICTC, 2013).

Dos 15 sorotipos de C. trachomatis, quatro (A, $\mathrm{B}, \mathrm{Ba}$ e C) são responsáveis pela infecção do tracoma (KOIZUMI et al., 2005), cujo único reservatório é o homem (MARIOTI; PASCOLINI; ROSE-NUSSBAUMER, 2009), que pode ser infectado de forma direta ou indireta. A infecção direta se dá pelo contato íntimo olho no olho, já a infecção indireta ocorre pelo compartilhamento de objetos contaminados com a secreção ocular (RAMESH et al., 2001).

Determina-se TF a forma como se apresenta a doença em sua fase inicial (ativa), caracterizando-se pela presença de cinco ou mais folículos na conjuntiva tarsal superior; o TI, por sua vez, apresenta-se com um pronunciado espessamento inflamatório da conjuntiva tarsal que obscurece mais da metade dos vasos tarsais profundos normais; já o TS, corresponde à presença de tecido fibroso (cicatricial) na conjuntiva tarsal. A forma clínica TT é assim denominada quando pelo menos um cílio arranha o globo ocular ou quando há evidência de recente remoção de cílios introvertidos. A forma mais grave, a CO, é a visível opacidade da pupila (OMS, 2015).

A Organização Mundial de Saúde (OMS) inclui o tracoma como uma doença tropical negligenciada, dadas as suas áreas de ocorrência (aquelas com presença de clima tropical) e o pouco interesse que recebe por parte dos governos e do mercado farmacêutico (OMS, 2003). Tendo em vista o objetivo de eliminação global do tracoma até 2020 (GET 2020), a OMS tem incentivado o seu mapeamento e endossado a SAFE Strategy, que envolve cirurgia para triquíase, antibioticoterapia, lavagem do rosto e saúde ambiental (EMERSON et al., 2006).

Embora a prevalência global do tracoma ativo tenha diminuído de 146 milhões em 1995 para 84 milhões em 2007, segundo a OMS, há países que ainda não começaram a mapear, alguns ainda estão em fase de programa ativo, enquanto outros estão próximo da eliminação (AAO, 2016). Hotez et al. (2008) estimaram que na América Latina 1,1 milhão de pessoas têm tracoma, representando 1,3\% da carga global, sendo o Brasil o país latino-americano com a situação mais crítica, com prevalência média de 5\% de tracoma folicular com variações de 1,5\% a 9\% nos 26 Estados e Distrito Federal (LUNA et al., 2016).

Por ser o Piauí um Estado cuja grande parte dos municípios apresenta baixo Índice de Desenvolvimento Humano (IDH) e baixas condições sanitárias (IBGE, 2010; OLIVEIRA, 2015), o que favorece a disseminação do tracoma, e tendo em vista a necessidade de atualização sobre a situação epidemiológica do tracoma no Estado, este estudo objetivou identificar a epidemiologia do tracoma no Piauí com enfoque para o número de casos positivos da doença, formas clínicas e locais de ocorrência.

\section{METODOLOGIA}

Trata-se de um estudo descritivo exploratório de abordagem quantitativa, realizado por meio de dados secundários de acesso restrito dos casos de tracoma diagnosticados no Estado do Piauí constantes no Sistema de Informação de Agravos de Notificação (SINAN). O Sinan abriga dados coletados por meio da busca ativa de tracoma em escolares em instituições educacionais públicas, onde o exame oftalmológico e casos positivos eram diagnosticados e imediatamente notificados. Casos positivos são também identificados em inquérito domiciliar quando da avaliação dos comunicantes.

A pesquisa desenvolveu-se noâmbito do Programa de Iniciação Científica e Tecnológica da Faculdade Integral Diferencial (Facid/DeVry), após autorização pela Secretaria de Estado da Saúde do Piauí (Sesapi) por meio da assinatura do Termo de Autorização Institucional (TAI) e Termo de Compromisso de Utilização de Dados (TCUD). Este estudo dispensou submissão ao Comitê de Ética em Pesquisa por não envolver seres humanos direta ou indiretamente como sujeitos.

Foram incluídos neste estudo, notificações de casos positivos de tracoma no Estado do Piauí, conforme consta no Sinan. Não houve determinação prévia de 
faixa etária, sexo, local de ocorrência ou forma clínica do tracoma, pois o próprio sistema informatizado organiza os dados nele contidos dentro das mencionadas categorias.

A coleta de dados se deu entre os meses de agosto a setembro de 2016, sendo os mesmos extraídos do Sinan, na sede da Sesapi em Teresina-PI, com supervisão de grupo técnico responsável pela guarda dos dados sobre tracoma no Estado. Os dados coletados foram então impressos para análise e tabulados no software Microsoft Office Excel 2010, para análise estatística (porcentagem simples), representação gráfica, segmentação das categorias de análise.

O IDH dos municípios piauienses foi obtido a partir da última compilação realizada pelo Programa das Nações Unidas para o Desenvolvimento no ano de 2010, para que se evidenciasse a relação com o baixo IDH e a ocorrência de casos positivos de tracoma (PNUD, 2010)

\section{RESULTADOS}

A busca de dados sobre tracoma no Piauí por meio do Sinan apresentou notificações positivas entre 2007 a setembro de 2016, por meio de inquérito escolar e domiciliar, em 34 municípios do Estado, sendo os mais afetados aqueles com baixo IDH e em sua maioria localizados na região Sudoeste do Estado, a exemplo dos municípios de Guaribas, Santa Filomena, Sebastião Leal, dentre outros. Tracoma não se apresentou como um agravo preocupante nos municípios situados nas regiões Norte e Sudeste do Estado (Figura 1).

Em um recorte histórico de nove anos, houve um total de 593 notificações positivas de tracoma no Piauí, observando-se aumentos e quedas bruscas no número de casos ao longo dos anos, sendo o ano de 2014 aquele com o maior número de notificações $(23,6 \%)$, com queda acentuada nos anos de 2015 e 2016 (7,1\% e $0,2 \%$, respectivamente). A maioria dos casos positivos foi identificada por meio de inquérito escolar (Tabela 1).
Tabela 1. Frequência dos casos positivos de tracoma no Piauí segundo tipo de inquérito 2007-Set 2016. Teresina, Piauí, Brasil, 2016

\begin{tabular}{ccccc}
\hline \multirow{2}{*}{ Ano } & \multicolumn{2}{c}{ Tipo de inquérito } & & \\
\cline { 2 - 3 } & $\begin{array}{c}\text { Escolar } \\
(\mathbf{n})\end{array}$ & $\begin{array}{c}\text { Domiciliar } \\
(\mathbf{n})\end{array}$ & Total (n) & $\%$ \\
\hline 2007 & 20 & - & 20 & 3,4 \\
2008 & 75 & & 75 & 12,6 \\
2009 & 66 & - & 66 & 11,1 \\
2010 & 38 & - & 38 & 6,4 \\
2011 & 106 & - & 106 & 17,9 \\
2012 & 67 & 7 & 74 & 12,5 \\
2013 & 27 & 6 & 33 & 5,6 \\
2014 & 127 & 13 & 140 & 23,6 \\
2015 & 40 & - & 40 & 6,7 \\
$2016 *$ & 1 & - & 1 & 0,2 \\
\hline Total & 567 & 26 & 593 & 100.0 \\
\hline Legenda: *mês de setembro. & & &
\end{tabular}

FONTE: SINAN 


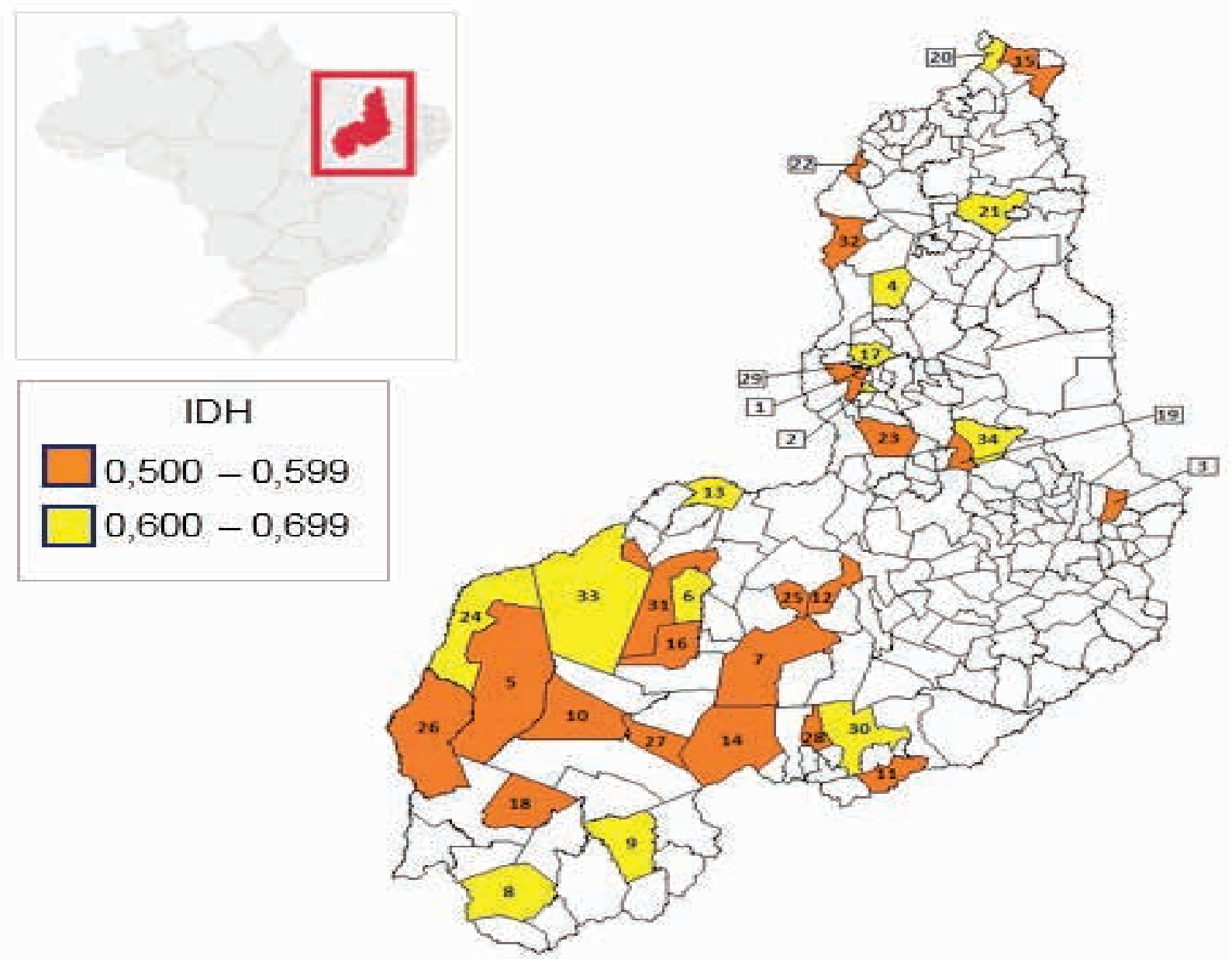

\begin{tabular}{|l|l|l|}
\hline 1 Agricolândia & 13 Guadalupe & 25 Rio Grande do Piauí \\
\hline 2 Água Branca & 14 Guaribas & 26 Santa Filomena \\
\hline 3 Alagoinha do Piauí & 15 Luis Correia & 27 Santa Luz \\
\hline 4 Altos & 16 Manoel Emídio & 28 São Braz do Piauí \\
\hline 5 Baixa Grande do Ribeiro & 17 Monsenhor Gil & 29 São Pedro do Piauí \\
\hline 6 Bertolina & 18 Monte Alegre do Piauí & 30 São Raimundo Nonato \\
\hline 7 Canto do Buriti & 19 Novo Oriente do Piauí & 31 Sebastião Leal \\
\hline 8 Corrente & 20 Parnaíba & 32 União \\
\hline 9 Curimatá & 21 Piripiri & 33 Uruçuí \\
\hline 10 Currais & 22 Porto & 34 Valença do Piauí \\
\hline 11 Dirceu Arcoverde & 23 Regeneração & \\
\hline 12 Flores do Piauí & 24 Ribeiro Gonçalves & \\
\hline
\end{tabular}

Figura 1. Municípios piauienses com notificações de casos positivos de tracoma de 2007 a Set 2016 por IDH. Teresina, PI. 2016 Fonte: Elaborado pelo Autor 
Tabela 2. Frequência dos casos positivos de tracoma no Piauí por município 2007-Set 2016. Teresina, Piauí, Brasil, 2016

\begin{tabular}{|c|c|c|c|c|c|c|c|c|c|c|c|c|}
\hline \multirow[t]{2}{*}{ Município } & \multicolumn{10}{|c|}{ Ano } & \multirow{2}{*}{ Total (n) } & \multirow{2}{*}{$\%$} \\
\hline & 2007 & 2008 & 2009 & 2010 & 2011 & 2012 & 2013 & 2014 & 2015 & 2016 & & \\
\hline Agricolândia & - & - & - & - & - & - & - & - & - & 1 & 1 & 0,2 \\
\hline Água Branca & - & - & 2 & - & - & - & - & - & - & - & 2 & 0,3 \\
\hline Alagoinha do Piauí & & $\cdot$ & 3 & - & 1 & - & - & - & - & - & 4 & 0,7 \\
\hline Altos & 20 & 7 & - & - & - & 1 & - & - & - & - & 28 & 4,7 \\
\hline Baixa Grande do Ribeiro & - & - & - & 15 & 3 & - & 7 & - & - & - & 25 & 4,2 \\
\hline Bertolínia & - & - & - & - & 21 & - & - & - & - & - & 21 & 3,5 \\
\hline Canto do Buriti & - & - & - & - & - & - & - & 5 & - & - & 5 & 0,8 \\
\hline Corrente & - & - & - & - & - & - & - & 19 & - & - & 19 & 3,2 \\
\hline Curimatá & & 39 & - & - & 1 & - & 2 & - & - & - & 42 & 7,1 \\
\hline Currais & - & - & - & - & 25 & 4 & - & - & - & - & 29 & 4,9 \\
\hline Dirceu Arcoverde & & - & - & - & - & 1 & - & - & - & - & 1 & 0,2 \\
\hline Flores do Piauí & - & - & - & - & - & - & - & 1 & - & - & 1 & 0,2 \\
\hline Guadalupe & - & - & - & - & - & 3 & 12 & 16 & 7 & - & 38 & 6,4 \\
\hline Guaribas & - & - & 23 & - & - & 8 & 4 & 13 & - & - & 48 & 8,1 \\
\hline Luís Correia & - & - & - & - & - & & & 36 & - & - & 36 & 6,1 \\
\hline Manoel Emídio & - & - & - & - & - & 8 & 1 & - & - & - & 9 & 1,5 \\
\hline Monsenhor Gil & - & $\cdot$ & - & - & - & - & - & - & 5 & - & 5 & 0,8 \\
\hline Monte Alegre do Piauí & - & - & - & - & - & 13 & - & 7 & - & - & 20 & 3,4 \\
\hline Novo Oriente do Piauí & - & - & - & - & - & - & - & 2 & 1 & - & 3 & 0,5 \\
\hline Parnaíba & - & - & 8 & - & - & - & - & 19 & - & - & 27 & 4,6 \\
\hline Piripiri & - & $\cdot$ & - & - & - & - & - & - & 1 & - & 1 & 0,2 \\
\hline Porto & - & $\cdot$ & 13 & - & 5 & - & - & 2 & - & - & 20 & 3,4 \\
\hline Regeneração & - & - & - & - & - & - & 1 & - & - & - & 1 & 0,2 \\
\hline Ribeiro Gonçalves & - & - & - & 23 & - & 3 & 3 & - & - & - & 29 & 4,9 \\
\hline Rio Grande do Piauí & - & $\cdot$ & - & - & - & 2 & 3 & - & - & - & 5 & 0,8 \\
\hline Santa Filomena & - & 26 & - & - & 5 & - & - & 18 & - & - & 49 & 8,3 \\
\hline Santa Luz & - & - & 12 & - & - & - & - & - & - & - & 12 & 2,0 \\
\hline São Braz do Piauí & - & - & 1 & - & - & - & - & - & - & - & 1 & 0,2 \\
\hline São Pedro do Piauí & - & - & - & - & 8 & - & - & 2 & - & - & 10 & 1,7 \\
\hline São Raimundo Nonato & - & - & 3 & - & - & - & - & - & - & - & 3 & 0,5 \\
\hline Sebastião Leal & - & - & - & - & 37 & - & - & - & - & - & 37 & 6,2 \\
\hline União & - & - & - & - & - & 1 & - & - & - & - & 1 & 0,2 \\
\hline Uruçuí & - & - & - & - & - & 30 & - & - & 26 & - & 56 & 9,4 \\
\hline Valença do Piauí & - & 3 & 1 & - & - & - & - & - & - & - & 4 & 0,7 \\
\hline Total & 20 & 75 & 66 & 38 & 106 & 74 & 33 & 140 & 40 & 1 & 593 & 100,0 \\
\hline
\end{tabular}

Fonte: SINAN 
Uruçuí, com população de 20.149 habitantes, foi o município com o maior número de casos notificados positivos de tracoma $(9,4 \%)$, seguido por Santa Filomena $(8,3 \%)$ com população de 6.096 , Guaribas $(8,1 \%)$ com população de 4.401 e Curimatá (7,1\%), que possui 10.761 habitantes (BRASIL, 2016; IBGE, 2010) (Tabela 2).
Em relação às formas clinicas do tracoma, houve predominância de casos de tracoma folicular (TF) (99\%), ocorrendo ao longo dos nove anos (2007-2016). No mesmo recorte histórico, foram notificados sete casos de tracoma inflamatório intenso (TI) (1\%). Apenas um caso de tracoma cicatricial (TS) foi notificado (em 2011). As formas clínicas triquíase tracomatosa (TT) e opacidade corneana (CO) não foram notificados (Figura 2).

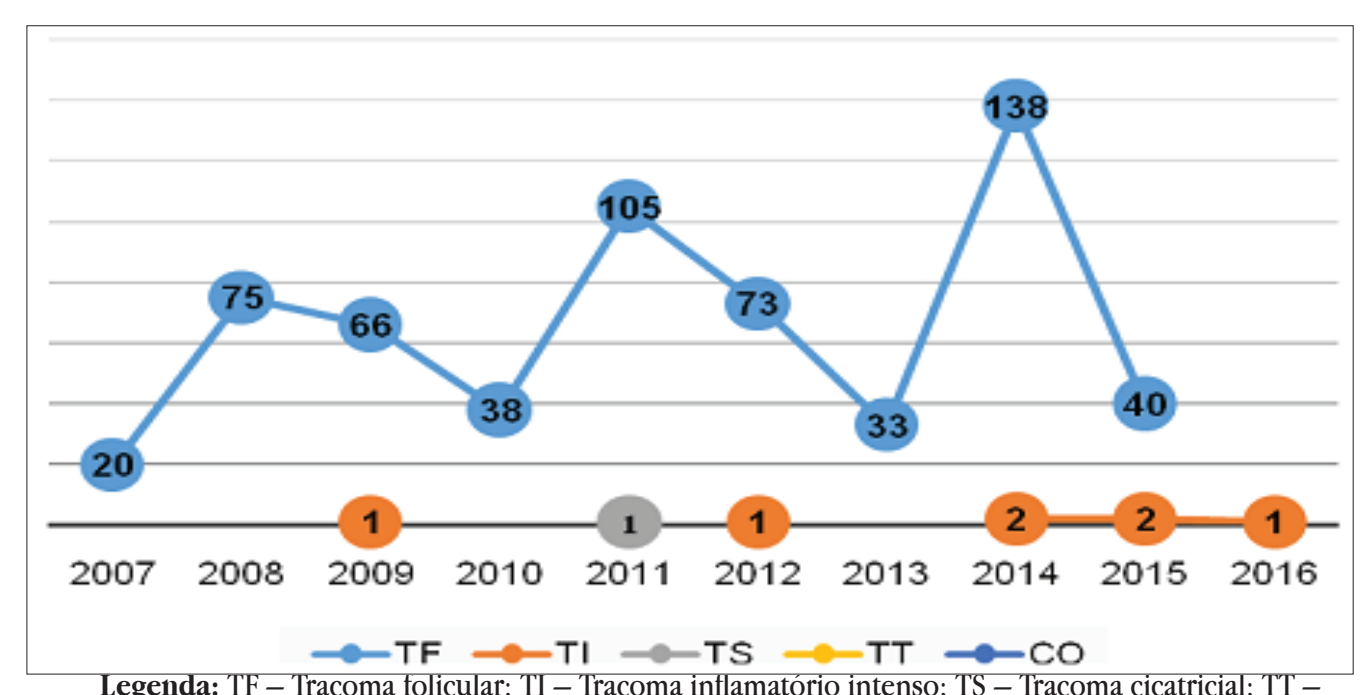

Legenda: TF - Tracoma folicular; TI - Tracoma inflamatório intenso; TS - Tracoma cicatricial; TT Triquíase tracomatosa; $\mathrm{CO}$ - Opacidade corneada

Figura 2. Frequência dos casos positivos de tracoma por forma clínica 2007-Set 2016. Teresina, Piauí, 2016 Fonte: Elaborado pelo autor

\section{DISCUSSÃO}

O tracoma não existia dentre os americanos nativos do continente, mas foi trazida pelos colonizadores. No Brasil, a doença foi trazida por colonos e imigrantes, com os primeiros focos surgindo no século XVIII no nordeste nas regiões onde hoje se constituem os Estados do Ceará e Maranhão. Porém, rapidamente a doença se alastrou por todo o Brasil (BRASIL, 2014).

Um dos nove Estados do nordeste, o Piauí possui 224 municípios e população estimada em 3.118.360 habitantes, sendo 1.067.401 residentes na zona rural. O Estado apresenta um IDH médio $(0,646)$, segundo dados compilados PNUD em 2010 (PNUD, 2010).

Em 2006, no último inquérito nacional de prevalência de tracoma em escolares, realizado no Piaú, pela Secretaria de Vigilância em Saúde do Ministério da Saúde (SVS/MS), identificou-se a prevalência de tracoma folicular (TF) de 4,4\% no Estado, com variações entre 0 e $23 \%$ nos municípios. Segundo dados do inquérito nacional, prevalência acima de $10 \%$ foi identificada nos municípios de Porto, São Pedro do Piauí, Guadalupe, Valença do Piauí, Manoel Emídio, Guaribas, Pau D’Arco do Piauí, Santa Luz, Monte Alegre do Piauí, Santa Luz, Monte Alegre do Piauí, Sebastião Leal, Baixa Grande do Ribeiro e Santa Filomena (BRASIL, 2011; LUNA et al., 2016).

Fazendo-se uma análise comparativa dos dados sobre tracoma do último inquérito nacional de 2006 e daqueles disponibilizados pelo Sinan em inquéritos realizados pelo Estado (entre 2007 e 2016), observase que o número de municípios afetados pelo tracoma no Estado do Piauí reduziu-se sobremaneira, com prevalência acima de 10\% registrada apenas em Uruçuí, entre 2008 e 2010 (BRASIL, 2011).

Considerando-se os achados deste estudo devese dar atenção, portanto, ao município de Uruçuí, mas também aos casos de tracoma notificados em municípios onde a doença não havia sido identificada há anos e, a partir de 2012 ou nos anos seguintes foram notificados no Sinan, como o que se observou acontecer nos municípios de Agricolândia, Canto do Buriti, Corrente, 
Dirceu Arcoverde, Flores do Piauí, Guadalupe, Luís Correia, Manoel Emídio, Monsenhor Gil, Monte Alegre do Piauí, Novo Oriente, Piripiri, Regeneração, Rio Grande do Piauí e União (BRASIL, 2011; BRASIL, 2016).

É pertinente, portanto, conhecer a origem dos casos notificados e investigar de que forma o tratamento vem sendo conduzido, tendo em vista o estabelecido pela OMS: tratamento em massa na comunidade quando a prevalência supera $10 \%$ em crianças de $1-9$ anos de idade e tratamento extensivo à família como profilaxia em menores taxas de prevalência, além de doses subsequentes anuais, durante três anos (SOLOMON et al., 2006).

Em estudo que apresentou os casos de tracoma diagnosticados em inquéritos epidemiológicos no Piauí no período de 2007 a 2013, identificou-se não haver predominância de sexo, sendo $50,4 \%$ dos casos no sexo feminino e $49,6 \%$ no sexo masculino (OLIVEIRA, 2015). Porém, houve mudança nesse dado, conforme identificamos com a análise de dados do Sinan. Em um período que se inicia em 2007 e se estende até 2016, das 593 notificações positivas realizadas, 57\% casos ocorreram no sexo masculino e $43 \%$ no sexo feminino.

Analisando as faixas etárias acometidas, ressaltamos que nem todas as notificações realizadas apresentaram informação acerca da faixa etária dos indivíduos acometidos, razão pela qual se considerou para esta análise 565 indivíduos cuja informação estava disponível. Assim, identificou-se que tanto crianças como adolescente estão sob maior risco para infecção por $C$. trachomatis e, a partir dos cinco a nove anos, a tendência foi de redução do número de notificações. Na faixa etária de um a quatro anos, 38 casos $(6,7 \%)$ foram notificados ao longo dos nove anos. As faixas etárias mais acometidas foram entre cinco e nove anos, com 297 casos (52,6\%) e dez e 14 anos de idade, com 213 casos (37,7\%). Nos indivíduos com idade entre 15-19 anos, 13 casos (2,3\%) foram identificados e apenas dois casos de tracoma foram notificados na faixa etária de 20-34 e 35-49 anos, somando-se quatro casos $(0,7 \%)$.

Tendo em vista as formas clínicas do tracoma identificadas no Estado do Piauí, com a forma primária (TI) sendo a mais recorrente, é de suma importância conhecer as principais manifestações apresentadas por indivíduos acometidos (MELO et al., 2016). Assim, com base na classificação simplificada para o tracoma, elaborada pela OMS, há cinco formas clínicas para a doença, sendo as mesmas progressivas e de gravidade crescente. O grau de acometimento no indivíduo infectado depende, portanto, do tempo de exposição sem o devido tratamento (OMS, 2003).

É fato que a prevalência do tracoma está relacionada ao baixo IDH e a determinantes sociodemográficos, sendo indispensável a adoção de práticas de promoção de saúde que venham capacitar gestores e sociedade civil a adotar atitudes capazes de modificar a situação epidemiológica da doença com resultante melhora na qualidade de vida da comunidade (BRASIL, 2014). Essas atitudes envolvem não apenas a prevenção e controle, mas políticas de desenvolvimento e melhorias de condições de vida e saúde da população (LOPES, 2013).

As limitações encontradas durante o curso desta pesquisa consistiram na pouca disponibilidade de estudos sobre a situação epidemiológica do tracoma no Estado do Piauí. Tal fato aponta para a necessidade da realização de mais estudos sobre essa temática, tendo em vista a popularização do conhecimento e fornecimento de subsídios para o desenvolvimento e implementação de políticas públicas que contribuam para a eliminação do tracoma no Estado.

\section{CONCLUSÃO}

Analisando-se dados do Sinan nos últimos nove anos e comparando-os com dados do último inquérito nacional sobre o tracoma, houve observável redução no número de municípios afetados pelo tracoma no Piauí. Alguns municípios não haviam notificado casos positivos há mais de cinco anos e vieram a identificar a infecção por $C$. trachomatis nos últimos anos, razão pela qual merecem atenção.

Municípios localizados na região Sudoeste do Estado e que apresentam baixo IDH foram aqueles que maior presença de casos positivos de tracoma. Não obstante, cidades com IDH médio também notificaram tracoma em sua população, com a grande maioria dos casos sendo tracoma folicular, além de casos raros e isolados tracoma inflamatório intenso e apenas um caso de tracoma cicatricial.

O ano de 2014 foi o que apresentou maior 
incidência da doença sobre a população piauiense, sobretudo Luís Correia, litoral do Estado. Porém, no ano seguinte, Uruçuí volta a liderar como o município que apresenta o maior número de casos positivos de tracoma notificados, merecendo atenção.

\section{REFERÊNCIAS}

AMERICAN ACADEMY OF OPHTHALMOLOGY. Trachoma - Latin America. Washington: AAO, 2016. Disponível em: <https://www.aao.org/topic-detail/trachoma--latinamerica >. Acesso em: 2 dez. 2016.

BRASIL. Ministério da Saúde. Secretaria de Vigilância em Saúde. Manual de vigilância do tracoma e sua eliminação como causa de cegueira. Brasília: MS, 2014.

BRASIL. Ministério da Saúde. Secretaria de Vigilância em Saúde. Sistema nacional de vigilância em saúde: relatório de situação: Piauí. Brasília: MS, 2011.

BRASIL. Ministério da Saúde. Sistema de Sistema de Informação de Agravos de Notificação. Tracoma: notificações registradas: banco de dados. Brasília: 2016.

EMERSON, P.M.; BURTON, M.; SOLOMON, A.W.; BAILEY, R.; MABEY, D. The Safe strategy for trachoma control: using operational research for policy, planning and implementation. Bull World Health Org, v.84, n.8, p. 613-619, 2006.

HOTEZ, P.J.; BOTTAZZI, M.E.; FRANCO-PAREDES, C.; AULT, S.K.; PERIAGO, M.R. The neglected tropical diseases of Latin America and the Caribbean: a review of disease burden and distribution and a roadmap for control and elimination. PLoS Negl Trop Dis, v.9, n.1, p.1-11, 2008.

INSTITUTO BRASILEIRO DE GEOGRAFIA E ESTATÍSTICA. Censo populacional 2010. Rio de Janeiro: IBGE, 2010. Disponível em: <http://censo2010.ibge.gov.br/> . Acesso em: 6 dez. 2016.

INTERNATIONAL COALITION FOR TRACHOMA CONTROL. Práticas preferidas para administração em massa do Zithromax. Washington: ICTC, 2016.

KOIZUMI， I.K.; MEDINA, N.H.; D'AMARAL, R.K.K.; MORIMOTO, W.T.M.; CALIGARIS, L.S.A.;CHINEN, N. et al. Prevalência do tracoma em pré-escolares e escolares no município de São Paulo. Rev. Saúde Pública, v.39, n.6, p. 937-942, 2005.

LOPES, M. de F.C.; LUNA, E.J. de A.; MEDINA, N.H.;
CARDOSO, M.R.A.; FREITAS, H.S. de A.; KOIZUME, I.K. et al. Prevalência de tracoma entre escolares brasileiros. Rev. Saúde Pública, v.47, n.3, p.451-459, 2013.

LUNA, E.J.A.; LOPES, M.F.C.; MEDINA, N.H.; FAVACHO, J.; CARDOSO, M.R. Prevalence of trachoma in schoolchildren in Brazil. Ophthalmic Epidemiol, v.23, n.6, p.360-365, 2016.

MARIOTI, S.P.; PASCOLINI, D.; ROSE-NUSSBAUMER, J. Trachoma: global magnitude of a preventable cause of blindness. Br J Ophthalmol., v.93, n.5, p. 563-568, 2009.

MELO, M.C.B. de.; FALCÃO, T.M.L.; SILVA, J.A.M.; ROCHA, T.T. de A.; ANDRADE, A.R. de. Tracoma: atenção prestada pelos profissionais da estratégia saúde da família em uma área endêmica. Saúde Pesqui., v.9, n.2. p.273-281, 2016.

OLIVEIRA, M.D. Situação epidemiológica do tracoma entre estudantes da rede pública de municípios do Piauí. 2015.53f. Dissertação (Mestrado em Epidemiologia em Saúde Pública) - Escola Nacional de Saúde Pública Sergio Arouca, Teresina, 2015.

ORGANIZAÇÃO MUNDIAL DE SAÚDE. Report of the 2nd Global Scientific Meeting on Trachoma. Washington: WHO, 2003. Disponível em: <http://www. who.int/blindness/2nd\%20GLOBAL\%20SCIENTIFIC\%20 MEETING.pdf> . Acesso em: 4 dez. 2016.

ORGANIZAÇÃO MUNDIAL DE SAÚDE. WHO simplified trachoma grading system. Community Eye Health, v.17, n.52, p.1, 2015.

PROGRAMA DAS NAÇÕES UNIDAS PARA O DESENVOLVIMENTO. Desenvolvimento Humano e IDH. Brasília: PNUD, 2010. Disponível em: <http:// www.br.undp.org/content/brazil/pt/home/idh0.html > . Acesso em: 1 jan. 2017.

RAMESH, A.; KOVATS, S.; HASLAM, D.; SCHMIDT, E.; GILBERT, C.E.The impact of climatic risk factors on the prevalence, distribution, and severity of acute and chronic trachoma. PLoS Negl Trop Dis, v.7, n.11, p. 1-8, 2001.

SOLOMON, A.W.; ZONDERVAN, M.; KUPER, H.; BUCHAN, J.C.; MABEY, D.C.W.; FOSTER, A. Trachoma control: a guide for programme managers. Geneva: WHO, 2006.

Recebido em:08 de setembro de 2016 Aceito em: 22 de abril de 2017 